\title{
Face Recognition Using LTP Algorithm
}

\author{
Richa Sharma ${ }^{1}$, Rohit Arora ${ }^{2}$ \\ ${ }^{1} \mathrm{ECE} \& \mathrm{KUK}$ \\ ${ }^{2}$ Assistant Professor (ECE)
}

\begin{abstract}
These approaches utilize different features for face recognition purpose. The feature utilized for face recognition are shape, distance between two traits of face, texture features for face. Texture features are particularly susceptible to the resolution of images, when the resolution changes the calculated textures are not accurate. Texture features computed for low resolution face images does not provide better feature information. So there is a big issue in face recognition for low resolution images. EULBP (Equalized Uniform Local binary Pattern) has been implemented for the purpose of low resolution but it does not provide better results up to an extent. The purpose of the research is to improve the accuracy for the low resolution images. By analysing various approaches for face recognition there is need to develop a new approach which can provide better results using texture features for blurred images. We will use LTP algorithm. LTP partially solves the noise-sensitive problem by encoding the small pixel difference into a separate state
\end{abstract}

Keywords: Face Recognition, EULBP, LTP, FAR, FRR

\section{Introduction}

\subsection{Face Recognition}

Face plays an important role in the various social activities to identity and emotion capturing. The human has a remarkable memory to recognize the individual by seeing his face. A number of faces have been seen by a single individual in his daily life routine. The face images stored in human brain and the individual can recognise the individual easily. So it is easy process for a single individual to recognise a person if they meet after number of years. But the aging, emotions and conditions can affect the representation of the face plays a major role in our social intercourse in conveying identity and emotion. The human ability to recognize faces is remarkable. We can recognize thousands of faces learned

Throughout our lifetime and identify familiar faces at a glance even after years of separation. The skill is quite robust, despite large changes in the visual stimulus due to viewing conditions, expression, aging, and distractions such as glasses or changes in hairstyle.

\subsection{Challenges in Face Recognition}

- Scale: Scaling factor in the face recognition process plays a vital role. In the process of face recognition different algorithms have to be implemented for the processing of various facial images. Eigen faces have to be developed for face recognition process. The main advantage of Eigen faces is that query image has to be matched with different Eigen faces of the database images. So main issue of scaling is that the Eigen face that has been developed using different images must be closer part of the facial image. So in these images can be scaled by using different scaling factor.

- Variation in Poses: Second main challenge $n$ face recognition is variation of pose. A small variance in the pose of the person can affect the accuracy of algorithm. Variation in pose can affect orientation of face images.
- Variation in luminance: Third main challenge that appears in face recognition process is the luminance. Due to variation in the luminance the representation get varied from the original image. The person with same poses expression and seen from same viewpoint can be appear very different due to variation in lightening.

- Facial Expression: Another challenge that affect the accuracy of face recognition program is that it affect due to change occurred in the facial expression.

- Disguise: The major issue that occur in face recognition is due to disguise. Glasses hairstyle, makeup and beard can change the appearance of the person. Most of the researcher works on this issue to resolve the affects of disguise.

\subsection{Parameters Used}

FAR (false acceptance rate)- The false acceptance rate, or FAR, is the measure of the likelihood that the biometric security system will incorrectly accept an access attempt by an unauthorized user. A system's FAR typically is stated as the ratio of the number of false acceptances divided by the number of identification attempts.

FRR (false rejection rate) - The false rejection rate, or FRR, is the measure of the likelihood that the biometric security system will incorrectly reject an access attempt by an authorized user. A system's FRR typically is stated as the ratio of the number of false rejections divided by the number of identification attempts.

Comparison between FAR \& FRR- These days there is a lot of emphasize on FAR (False Acceptance Rate). It is good that clients are getting aware of these terms and asking the right questions before implementing a solution. However, FAR only provides half the information. When selecting a biometric solution, we need to find out what the False Rejection Rate (FRR) is at the said FAR. So when a biometric solution provider claims to have a very low FAR, it is very important to find out what is the FRR at this 'low' FAR.? Then depending upon the application one needs to evaluate whether the FAR \& FRR ratio is acceptable for the application. In a practical scenario a low FAR \& a high FRR

\section{Volume 4 Issue 12, December 2015}




\section{International Journal of Science and Research (IJSR) \\ ISSN (Online): 2319-7064}

Index Copernicus Value (2013): 6.14 | Impact Factor (2014): 5.611

would ensure that any unauthorized person will not be allowed access. It would also mean that the authorized people will have to put their finger on the device several times before they are allowed access. Therefore, it is good to have a very low FAR, but please remember that if this low FAR is coming at the cost of high FAR then the solution needs to be re-evaluated.

\section{Review of Literature}

Kyungnam Kim "Face Recognition using Principle Component Analysis" [1] in this paper author proposed a technique Principal Component Analysis for face recognition. Principal Component Analysis was one of the most successful techniques that have been used for feature extraction from face image recognition. PCA utilized the one dimensional vector for the development of co-variance matrix. PCA was a statistical method to reduce the large dimensionality of features sub spaces. In the face recognition the principal component analysis describe the co-variance matrix. That co-variance matrix is used to develop Eigen values for the feature vector. That Eigen values were used for the development of Eigen faces. On the basis of that Eigen face the face recognition was done by computing distance between query image Eigen face and database images Eigen faces.

Pong C. Yuen, J.H. Lai "Face representation using independent component analysis" [2] In this paper author purposed an approach independent component analysis that was used for face recognition, face recognition has been done on the basis of features computed by independent component analysis. Independent component analysis computed different independent features for the facial image. The major issues in the independent component analysis are that the components are independent but not all the components are orthogonal so that image which does not belongs to training dataset that can't be recognised by using independent component analysis approach. This paper purposed approaches that use least square solution that computes Householder Transformation to 1-dimenssional feature vector.

JianYang "Two Dimensional PCA: A New Approach to Appearance base face representation and Recognition" [3] in this paper author purposed a new technique 2-Dimensional Principal Component Analysis for facial image description. 2DPCA is mainly based on different 2-D image matrices that are required for feature extraction rather than that require 1-D in principal component analysis. From 2D metrics of the image covariance matrix has to be derived so that the Eigen values can be computed and used for feature extraction on the basis of those Eigen values. These Eigen values are used for construction of Eigen faces. Eigen faces are the basic features of facial image. 2DPCA technique is applied on different face image databases. Performance of purposed approach is always better than that of PCA in terms of accuracy..

Timo Ahomen, Abdenour Hadid and Matti Pietikainen "Face Description with Local Binary Patterns: Application to Face Recognition" [4] purposed a new approach for image representation by using the LBP (local binary pattern). The face image is divided into several regions. On each region of the face image a $3 * 3$ mask in applied that computes the binary patterns for each divided region. These binary patterns are concatenate to derive face descriptor. That face descriptor is the face feature that is known as texture features of facial image. This approach is mainly used for gray scale facial images. In this paper the accuracy of LBP for different dataset has been discussed.

Niloofar Amani1"A new approach for face image enhancement and recognition" [5] in this paper author purposed an approach that has been used for face recognition on the basis of histogram features. In this approach image enhancement is done on the basis of contrast enhancement. This contrast enhancement approach use different high frequency domain conversion and filtering of histograms. The image enhancement is done by converting the image in high frequency domain and digital filters have been implemented on the image. This approach use histogram equalization to enhance the global pixel values available in the image. After converting the image into high frequency domain Otsu method is implemented, that works on the principal of thresholding the value under the threshold limit have to be considered.

\section{Approaches Used}

Local Ternary Pattern: LTP partially solves the noisesensitive problem by encoding the small pixel difference into a separate state. How this research is supported in part by the Singapore National Research Foundation under its International Research Centre @ Singapore Funding Initiative and administered by the IDM Programmed Office. Ever, when the ternary code is split into a positive LBP code and a negative LBP code, it may result in a significant information loss. Furthermore, the positive and negative LBP histograms are strongly correlated, and hence a lot of redundant information may reside in those two histograms.

\section{Conclusion}

Face recognition is a process of one too many matching. In this process the user matches one person identity with several database images. Various approaches have been utilized for the purpose of face recognition. The feature utilized for face recognition are shape, distance between two traits of face, texture features for face. Texture features are particularly susceptible to the resolution of images, when the resolution changes the calculated textures are not accurate. Texture features computed for low resolution face images does not provide better feature information. By analysing various approaches for face recognition there is need to develop a new approach which can provide better results using texture features for blurred images. We used LTP, which is used

\section{References}

[1] Pong C. Yuen, J.H. Lai (2002) "Face representation using independent component analysis", in the journal of Elsevier B.V pattern recognition, vol. 35, issue 6, pp. 1247-1257, DOI 10.1016/S0031-3203(01)00101-7.

\section{Volume 4 Issue 12, December 2015}


[2] Yue ZENG, Dazheng FENG, Li XIONG (2011) “An Algorithm of Face Recognition Based on the Variation of 2DPCA, Journal of Computational Information Systems, vol. 7, issue 1, pp. 303-310.

[3] Niloofar Amani1, Asadollah Shahbahrami and Manoochehr Nahvi (2013) "A new approach for face image enhancement and recognition", International Journal of Advanced Science and Technology, Vol. 52,

[4] Demirel, Hasan, Ozcinar, Cagri and Gholamreza Anbarjafi (2010) "Satellite Image Contrast Enhancement Using Discrete Wavelet Transform and Singular Value Decomposition", IEEE Geoscience And Remote Sensing Letters, Vol. 7, No. 2.

[5] Wei Jin, Bin Li and Ming You (2012) "Feature Extraction Based on Equalized ULBP for Face Recognition", International Conference on Computer Science and Electronics Engineering, Vol. 2, pp. 532536, ISBN 978-1-4673-0689-8.

[6] Jiande Sun, Caiming Zhang and Hua Yan (2012) "LowResolution Face Recognition with Variable Illumination Based on Differential Images" International Conference on Intelligent Information Hiding and Multimedia Signal Processing (IIH-MSP), pp. 146-149, ISBN 9781-4673-1741-2.

[7] Deniz O., Castrillon M. and Hernández M. (2003), "Face recognition using independent component analysis and support vector machines" Pattern Recognition Letters, Volume 24, Issue 13, Pages 21532157, DOI 10.10160167-8655(03)00081-3.

[8] Dong-Ju Kim, Sang Heon Lee and Myoung Kyu Sohn (2013) "Face Recognition with Local Directional Patterns", International Journal of Security and Its Applications Vol. 7, No. 2.

[9] Georghiades, A.S. and Belhumeur, P.N. and Kriegman, D. J. (2001) "From Few too Many: Illumination Cone Models for Face Recognition under Variable Lighting and Pose" IEEE Trans. Pattern Anal. Mach. Intelligence, vol. 23, 6, pp. 643-660.

[10] Grgic, Mislav. Delac, Kresimir and Grgi, Sonja (2011) "SC face surveillance cameras face", Multimed Tools Appl, vol 51, pp.863-879. DOI 10.1007/s11042-009041.

[11]Huang, Gary B., Ramesh, Manu, Berg, Tamara and Learned-Miller, Erik (2007) "Labeled Faces in the Wild: A Database for Studying Face Recognition in Unconstrained Environments" University of Massachusetts, Amherst, Technical Report, Pp. 07-49.

[12] Hae Min Moon "The LDA-based Face Recognition at a Distance using Multiple Distance Image”, International Conference on Innovative Mobile and Internet Services in Ubiquitous Computing, pp. 249-255, DOI 10.1109/IMIS.2013.50 